\section{Análisis cefalométrico de Holdaway del perfil facial en adultos peruanos}

Holdaway Cephalometric analysis of soft tissue profile in peruvian adults

\section{Resumen}

El objetivo de este estudio consistió, en determinar las características del perfil blando en adultos, utilizando las medidas lineales y angulares del análisis de Holdaway, y al mismo tiempo compararlas con las normas establecidas para la raza caucásica. La muestra estuvo comprendida por 40 sujetos adultos (20 hombres y 20 mujeres) que no habían recibido tratamiento ortodóncico, cuyas edades estuvieron comprendidas entre los 18 y 30 años. Todos los sujetos gozaban de buena salud y presentaban una oclusión normal. Para realizar este estudio se tomaron radiografías cefalométricas de perfil en oclusión. Los resultados indicaron que en la mayoría de los valores lineales y angulares se encontraron diferencias significativas en relación con los valores reportados por Holdaway. El ángulo " $\mathrm{H}$ " en la muestra peruana fue $8.45^{\circ}$ mayor que el referido por Holdaway para la raza caucásica.
Tomás Oriel Orellana Manrique'; Luciano Soldevilla Galarza'; Pedro Ballona Chambergo ${ }^{2}$; Martín Orellana Manrique ${ }^{3}$; Iván Calderón Cortez ${ }^{4}$

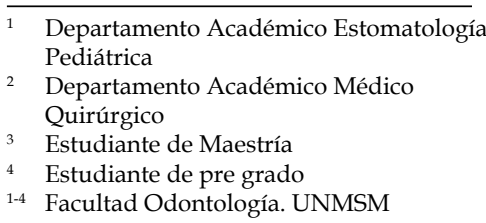

\section{Correspondencia:}

Mg. Oriel Orellana Manrique

Av. Diez Canseco 150-304

Miraflores. Lima - Perú.

e-mail: torellanam@unmsm.edu.pe

Palabras clave: Cefalometría. Perfil Facial. Análisis de Holdaway. Oclusión normal.

\begin{abstract}
The purpose of this study was to determine characteristics of soft-tissue profile in peruvian adults using Holdaway linear and angular measurements. It was also the purpose of this study to compare the collected data with the Caucasian norms of Holdaway. The sample consisted of 40 untreated adults ( 20 males and 20 females) between 18 to 30 ages. The subjects selected were in good health and had normal occlusion. Lateral cephalogram $\mathrm{x}$-rays were taken with the teeth in occlusion. The findings indicated that the most of linear and angular values were significantly different from those of the Holdaway analysis. The angle " $\mathrm{H}$ " in the Peruvian sample was $8.45^{\circ}$ more than that of the American. Caucasian group.
\end{abstract}

Key words: Cephalometry. Facial profile. Holdaway analysis. Normal occlusion.

\section{Introducción}

En una sociedad donde la belleza forma parte de un conjunto de cualidades personales, la estética facial juega un papel cada vez más importante en la vida de los individuos de todos los niveles sociales y económicos.

Angle $^{1}$, desde 1907, sugería que si los dientes se encontraban en una oclusión óptima, el resultado sería una buena armonía facial. Apuntó que el estudio de la ortodoncia estaba estrechamente relacionado con el arte en lo que se refiere a las proporciones de la cara, y la boca, era el factor más potente en exaltar o destruir la belleza y el carácter de la misma. También observó que la armonía-balance de la cara y forma-belleza de la boca de- pendían de la relación oclusal de los dientes. Concluyó que el complemento de todos los dientes en oclusión normal es esencial para las proporciones de cualquier cara.

El perfil de los tejidos blandos juega una parte importante en las consideraciones ortodóncicas. ${ }^{8}$ Cuando corregimos maloclusiones, traemos mejores cambios en la apariencia facial. Los objetivos más importantes de la ortodoncia son el de lograr una oclusión funcional y una estética dentofacial. Algunas decisiones terapéuticas son tomadas en función de mejorar o al menos no comprometer el perfil facial del paciente.

Todos los análisis cefalométricos del perfil de los tejidos blandos, presen- tan patrones de normalidad a partir de estudios realizados en grupos de individuos de raza caucásica, $3,4,5,6,12$ con excelentes oclusiones; los cuales constituyen parámetros de comparación cuando realizamos la evaluación cefalométrica de nuestros pacientes. Sin embargo, sabemos que existen diferencias entre las características anatómicas de las diferentes razas. ${ }^{7,10}$ Es por esta razón que se hace necesario, encontrar un perfil cefalométrico de nuestra población, de acuerdo a sus características propias.

El objetivo del presente estudio es de establecer los parámetros de normalidad de los tejidos blandos en una población peruana con excelente oclusión, según el análisis cefalométrico de los tejidos blandos de Holdaway.5,13 


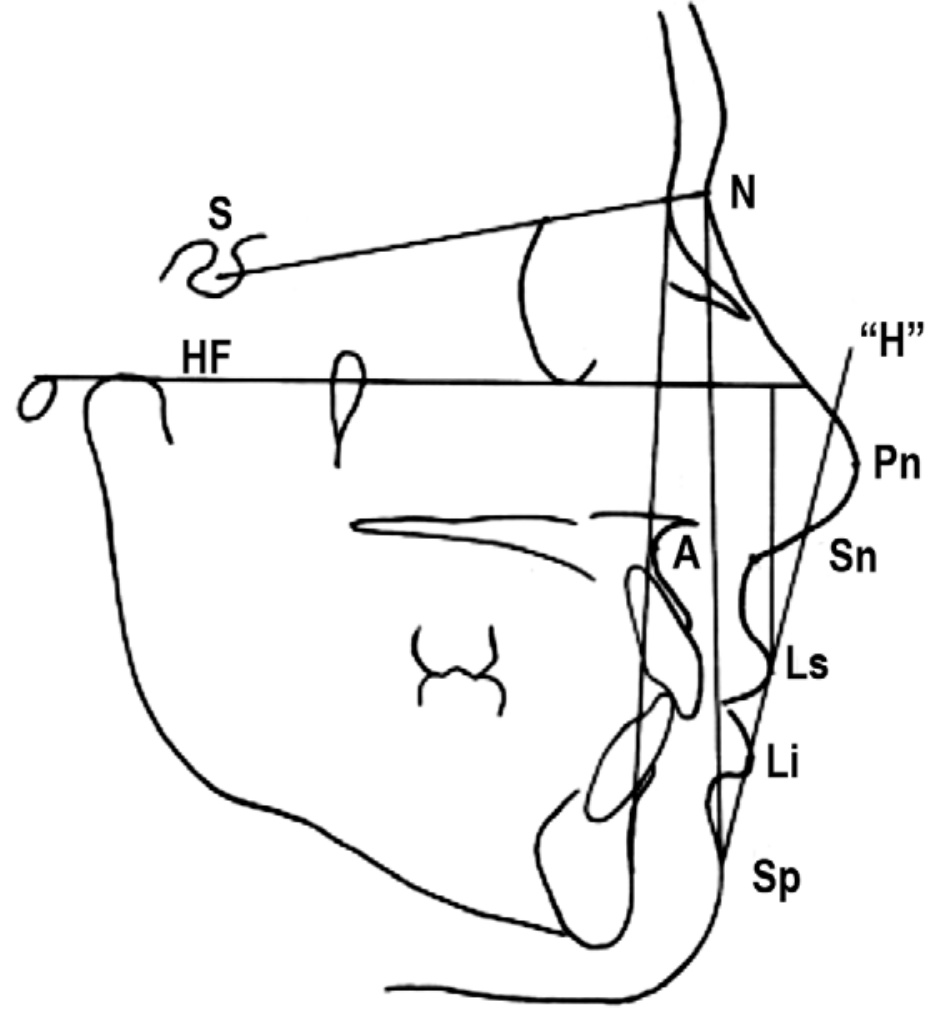

\section{ANÁLISIS DE HOLDAWAY}

Holdaway, en 1983, propuso un análisis cefalométrico de los tejidos blandos para el diagnóstico, así como para determinar los cambios en el perfil facial inducidos por el crecimiento y tratamiento. En su análisis, establece 11 parámetros para estudiar el perfil facial:

1. El Angulo facial de tejidos blandos (HF/Nb-Spb)

Es el ángulo formado por el plano de Frankfort (HF) con el plano Nasión blando-Suprapogonion blando).

Norma: $91^{\circ}$

Desviación Estándar: $7^{\circ}$

Interpretación: Localiza al mentón de tejidos blandos en el sentido ánteroposterior. Los valores mayores a la norma indican un perfil prognático(cóncavo), en donde la mandíbula se encuentra más adelantada; los valores menores a la norma indican un perfil retrognático (convexo) en donde la mandíbula se encuentra en una posición más retruida. Fig. 1.

\section{La Prominencia nasal (HF/Ls-Pn)}

Es la distancia que existe entre el punto pronasal(Pn) y una perpendicular a Frankfort que pasa por el punto Labial superior (Ls). esta medida.
4. Distancia Subnasal a la línea H (Sn / Spb-Ls)

Es la distancia que existe entre subnasal (Sn) y la línea H (Spb-Ls).

Norma: $5 \mathrm{~mm}$.

Desviación Estándar: 2 mm.

Interpretación: Determina el balance entre el surco labial, el labio superior y el pogonion blando. Una medida aumentada indica que el suprapogonion está ubicado muy atrás (clase II), que el labio superior es grueso, o que el surco es profundo. Una medida disminuida indica que el suprapogonion se encuentra adelantado (clase III), que el labio es delgado o que el surco es poco profundo.

5. Convexidad del perfil esquelético (A/N-Pg)

Es la distancia que existe entre el punto A y el plano facial (N-Pg). Cuando el punto A se encuentra por delante del plano facial, el valor es positivo. Si el punto A se encuentra por detrás del plano facial, el valor es negativo.

Norma: $0 \mathrm{~mm}$.

Desviación Estándar: $2 \mathrm{~mm}$.

Interpretación: Indica la ubicación ánteroposterior de la maxila en relación al perfil esquelético. Los valores mayores a la norma indican un patrón esquelético de clase II y los valores negativos indican un patrón esquelético de clase III. Esta no es una medida de tejidos blandos, pero se encuentra directamente relacionada con una posición armónica del labio superior y el perfil.

6. Grosor de la base del labio superior

Se mide a partir de $3 \mathrm{~mm}$. Por debajo del punto A hacia la máxima concavidad del surco labial (SLs).

Norma: $15 \mathrm{~mm}$.

Desviación Estándar: No establecida.

Interpretación: Indica la forma o el contorno del surco labial superior, pudiéndose modificar por el grosor del labio o por la posición ánteroposterior de los incisivos superiores. Esta medida es muy útil para determinar si se deben o no retraer los incisivos. $\mathrm{Si}$ no se considera esta medida se puede retraer demasiado el labio aplanando completamente el surco labial. Hay que tener en cuenta que en pacientes con tercios medios disminuidos el labio se engrosa, pudiendo aumentar
Interpretación: Mide el grosor de la base del labio superior. Esta medida es útil al compararla con el grosor del labio superior que se encuentra por encima de la corona de los incisivos a nivel del borde bermellón, ya que su relación determina la cantidad de tensión o incompetencia labial que presenta el paciente.

7. Tensión del labio superior (Pr-Ls)

Es la distancia que existe entre el punto Prosthion (Pr) y el punto Labial superior (Ls). 
Norma: 13 - $14 \mathrm{~mm}$.

Desviación Estándar: No establecida.

Interpretación: Indica el grosor del labio superior. Una altura vertical excesiva, puede provocar un adelgazamiento de más de $1 \mathrm{~mm}$. Cuando el grosor del labio en el borde bermellón es mayor al grosor de la base, generalmente se identifica una falta de crecimiento vertical.

\section{Angulo $\mathrm{H}$}

Es el ángulo formado por la línea $\mathrm{H}$ (Pgb-Ls) y el plano facial de tejidos blandos (Nb-Pgb)

Norma: $10^{\circ}$ cuando la convexidad mide $0 \mathrm{~mm}$.

Desviación Estándar: No establecida.

Sin embargo, las medidas de $7^{\circ}$ a $15^{\circ}$ están dentro del rango adecuado. Idealmente, conforme la convexidad aumenta el ángulo $\mathrm{H}$ también debe de aumentar.

Interpretación: Este ángulo mide la prominencia del labio superior en relación con los demás tejidos blandos del perfil. Se debe de considerar que la posición del mentón puede alterar de manera importante este ángulo.

\section{Labio inferior a la línea $\mathrm{H}$}

Es la distancia en milímetros que existe entre el punto labial inferior $(\mathrm{Li})$ y la línea $\mathrm{H}$ (Spb-Ls).

Norma: de 0 a $0.5 \mathrm{~mm}$.

Desviación Estándar: No establecida.

Los valores de $1 \mathrm{~mm}$ por detrás de la línea $\mathrm{Ho} 2 \mathrm{~mm}$. por delante son considerados como un rango aceptable.

Interpretación: Esta medida indica la posición del labio inferior en relación a los demás componentes del perfil blando.

\section{Surco inferior a la línea $H$}

Es la distancia entre el surco del labio inferior (Sli) y la línea H (Spb-Ls).

Norma: no se especifica.

Desviación Estándar: No establecida.

Interpretación: Determina el contorno del surco del labio inferior. Este contorno debe guardar una armonía con respecto al contorno del surco del labio superior. Además, es un buen indicador de que también se manejó la inclinación axial de los incisivos inferiores. Los procedimientos de nivelación con arcos redondos pueden provocar una proinclinación de los incisivos, exagerando el surco mentolabial. Por otro lado, si se retroinclinan los incisivos demasiado, el labio pierde su forma.

11.Grosor del mentón de tejidos blandos (Sp-Spb)

Es la distancia en milímetros entre el plano facial esquelético (N-Spb) y el plano facial de tejidos blandos(Nb$\mathrm{Spb})$ medido a nivel de Suprapogonion.

Norma: De 10 a 12 mm.

Desviación Estándar: No establecida.

Interpretación: Indica el grosor del tejido blando del mentón. Cuando se tienen medidas muy aumentadas se debe cuidar la inclinación de los incisivos ya que se puede perder el soporte labial y dejar un perfil muy cóncavo. Es decir, hay que tener cuidado y no retroinclinar demasiado los incisivos.

\section{Material y métodos}

La muestra estuvo conformada por las radiografías cefalométricas de perfil de

20 varones y de 20 mujeres, adultos comprendidos entre los 18 y los 30 años de edad, estudiantes de Odontología de la Facultad de Odontología de la Universidad Nacional Mayor de San Marcos, que fueron previamente seleccionados mediante un examen clínico y con sus modelos de estudio. La muestra presentaba una oclusión normal y un perfil agradable. En cada caso se tomaron radiografías cefalométricas de perfil con el equipo radiográfico de la Facultad de Odontología, luego se confeccionó el cefalograma respectivo y se hizo el análisis de Holdaway en forma manual.

\section{Resultados}

Se determinó la media así como la desviación estándar y se aplicó la prueba estadística " $\mathrm{t}$ " de student para un nivel de confianza P:0.05, a fin de determinar el grado de significancia en cada uno de los parámetros. En el parámetro $\mathrm{N}^{\circ} 10$, Holdaway no ha establecido la norma, por lo que no se pudo aplicar la " $\mathrm{t}$ " de student. (Tabla 1).

Tabla 1.- Valor promedio, desviaciones estándar $\mathrm{y}$ " $t$ " de student de la muestra peruana.

\begin{tabular}{|c|c|c|c|c|c|c|c|c|}
\hline \multirow{2}{*}{$\begin{array}{l}\text { Medidas } \\
\text { Cefalomé- } \\
\text { tricas }\end{array}$} & \multirow{2}{*}{$\begin{array}{l}\text { Unidad } \\
\text { de } \\
\text { medida }\end{array}$} & \multicolumn{2}{|c|}{ Holdaway } & \multicolumn{2}{|c|}{ Muestra } & \multirow{2}{*}{$\begin{array}{c}\mathrm{t} \\
\text { calc. }\end{array}$} & \multirow{2}{*}{$\begin{array}{c}\text { t. } \\
\text { Tabla }\end{array}$} & \multirow[t]{2}{*}{ Decisión } \\
\hline & & Media & $\overline{\mathrm{DE}}$ & Media & $\mathrm{DE}$ & & & \\
\hline 1 & Grados & 91 & 7 & 92.63 & 2.8 & 3.7 & 2.021 & Significativo \\
\hline 2 & $\mathrm{~mm}$ & 19 & 5 & 16.84 & 2.19 & -6.35 & 2.021 & Significativo \\
\hline 3 & $\mathrm{~mm}$. & 3 & 1 & 4.5 & 1.06 & 9.37 & 2.021 & Significativo \\
\hline 4 & $\mathrm{~mm}$. & 5 & 2 & 7.62 & 2.28 & 7.27 & 2.021 & Significativo \\
\hline 5 & $\mathrm{~mm}$. & 0 & 2 & 2.11 & 2.34 & 5.7 & 2.021 & Significativo \\
\hline 6 & $\mathrm{~mm}$. & 15 & - & 15.86 & 1.7 & 3.3 & 2.021 & Significativo \\
\hline 7 & $\mathrm{~mm}$ & 13 a 14 & - & 13.88 & 2.02 & 1.22 & 2.021 & No Significativo \\
\hline 8 & Grados & 10 & - & 18.45 & 3.77 & 14.32 & 2.021 & Significativo \\
\hline 9 & $\mathrm{~mm}$ & 0 a 0.5 & - & 1.71 & 1.78 & 5.21 & 2.021 & Significativo \\
\hline 10 & $\mathrm{~mm}$. & $\begin{array}{c}\text { No } \\
\text { estable- } \\
\text { cido }\end{array}$ & - & 4.07 & 1.6 & - & - & - \\
\hline 11 & $\mathrm{~mm}$. & 10 a 12 & - & 13.95 & 2.04 & 9.83 & 2.021 & Significativo \\
\hline
\end{tabular}

\section{Discusión}

El Angulo Facial de tejidos blandos ( $\mathrm{HF} / \mathrm{Nb}-\mathrm{Spb}$ ) fue ligeramente mayor en la muestra peruana que la establecida por Holdaway, indicando una tendencia hacia un perfil cóncavo.

La Prominencia Nasal de la muestra de estudio fue ligeramente más pequeña que la reportada por Holdaway.
La Profundidad del Surco del Labio Superior fue más marcado en la muestra peruana que en la caucásica, que indicaría una protrusión de los incisivos superiores.

La Distancia Subnasal a la línea " $\mathrm{H}$ " fue mayor en la muestra peruana que en la caucásica, lo que indicaría que existe protrusión de los incisivos superiores. 
El Punto Cefalométrico "A" se encontró más adelantado respecto al Plano Facial (N-Pg) en la muestra peruana que en la caucásica, que indicaría una cierta protrusión del Maxilar Superior.

La Tensión del Labio Superior de la muestra peruana estuvo dentro de los parámetros de Holdaway.

El Angulo " $\mathrm{H}$ " de la muestra peruana fue mucho mayor que el reportado por Holdaway, debido probablemente a la protrusión del Maxilar Superior que da como consecuencia una posición más adelantada del Labio Superior.

El Labio Inferior se encontró más adelantado en la muestra peruana que en la reportada por Holdaway, debido a una posible protrusión de los incisivos inferiores. ${ }^{7,10}$

La Profundidad del Surco Inferior en relación con la línea " $\mathrm{H}$ " de la muestra no se pudo comparar con el de la caucásica debido a que Holdaway no ha reportado los valores de normalidad.

El Grosor del Mentón de los Tejidos Blandos fue mayor en la muestra peruana que en la caucásica, lo que determinaría un perfil más cóncavo debido a un mentón de los tejidos blandos más marcado.

Al término del estudio se llegó a las siguientes conclusiones:

1. Los valores cefalométricos del perfil facial según el análisis de Holdaway en la muestra de la población peruana fueron mayores que el de la población caucásica y mostraron diferencias estadísticas significativas excepto para la Tensión del Labio Superior.

2. El valor del ángulo " $\mathrm{H}^{\prime}$, parámetro principal del análisis de Holdaway, fue mucho mayor en la muestra de la población peruana que en la caucásica, debido probablemente a la Protrusión del Maxilar Superior.

3. La posición más adelantada del Labio Inferior encontrado en la muestra peruana reflejaría una protrusión de los incisivos inferiores reportados en otros estudios.

\section{Agradecimiento:}

Al CSI de la UNMSM por el apoyo financiero para la ejecución del proyecto.

\section{Referencias bibliográficas}

1. Angle E.H. Malocclusion of the teeth. $7^{\circ}$ ed. Philadelphia. SS White Dental Mfg. Co. 1907. Citado por: Leon M.E, Arce R.M, y Espinoza M.A. Medidas cefalométricas en mujeres con características faciales agradables. Rev Colombia Médica. 2001;2(3):145-51.

2. Barrer J.G. y Ghrafari J. Silhouette profile in the assessment of the facial esthetics, a comparison of cases treated with various orthodontic applicances. AJODO. 1985;87:385-391

3. Bishara S.,Treder y Jakobsen J. Facial and dental changes in adulthood. AJODO. 1994 august;175-186
4. Burstone C.J. Integumental contour and extension patterms. Angle Orthodontist 1959;29:93-104

5. Holdaway R.A. A soft tissue cephalometric analisis and its use in orthodontic treatment planning AJODO. 1983;84:1-28

6. Mamandras A. Growth of lips in two dimensions, a serial cephalometric study. AJODO. 1984 Jul;61-66.

7. Orellana T. Estudio Comparativo de la posición del incisivo inferior en la clase I y en la clase II división 1. [Tesis de Maestría en Estomatología]. U.N.M.S.M. Lima, 1998

8. Proffit W. Ortodoncia. Teoría y Práctica, 2da edición. Madrid: Mosby Doyma Libros. 1994:149-150

9. Rains M. y Nanda R. Soft tissue changes asociated with maxillary incisor retraction. AJODO. 1982 June;481-488

10. Torres G. Patrón Cefalométrico de una población peruana según el análisis cefalométrico de Steiner. [Tesis para optar el título de Cirujano Dentista] U.N.M.S.M. Lima, 2002

11. Viazis A. Atlas deOrtodoncia. Principios y Aplicaciones clínicas. Buenos Aires: Médica Panamericana S.A.;1995:41-49

12. Walman B. Changes in lip contour with maxillary incisor retraction". Angle Orthodontist 1982;52:129-131

13. Zamora C. Atlas de cefalometría, análisis clínico y práctico. Colombia: Actualidades Médico Odontológicas Latinoamérica C A (AMOLCA); 2003:87-101

Recibido : 22-04-2007

Aceptado para publicación: 25-05-2007

\section{SEMINARIO - TALLER DE INVESTIGACIÓN 2007}

Se invita a los miembros integrantes del Instituto de Investigación Estomatológica y a toda la comunidad odontológica en general a participar en el Seminario - taller:

“DEFINIENDO NUEVAS LÍNEAS DE INVESTIGACIÓN EN SALUD BUCAL"

Organizado por el Instituto de Investigación Estomatológica de la Facultad de Odontología, a llevarse a cabo el 2 y 3 de agosto del presente año. 